\section{The air rifle: a dangerous weapon}

Airgun injuries are common among children. Though several reports of eye and soft-tissue injuries have been published, intra-abdominal injuries are apparently rare. I report a case of gastric perforation by an airgun pellet, which exemplifies the potentially lethal injuries that these weapons can inflict. Though the current laws restricting the possession and use of air weapons are adequate, no effort is made to educate either children or parents in the potential danger of these "toys."

\section{Case report}

A 12-year-old boy presented with a pellet wound in the abdomen after being shot with a $\cdot 22$ calibre air rifle from a distance of $4 \cdot 3 \mathrm{~m}$. Examination disclosed an entry wound over the left costal margin but no signs of haemorrhage or peritoneal irritation. Radiographs in two planes showed the pellet lying posteriorly in the right upper abdomen (figure).
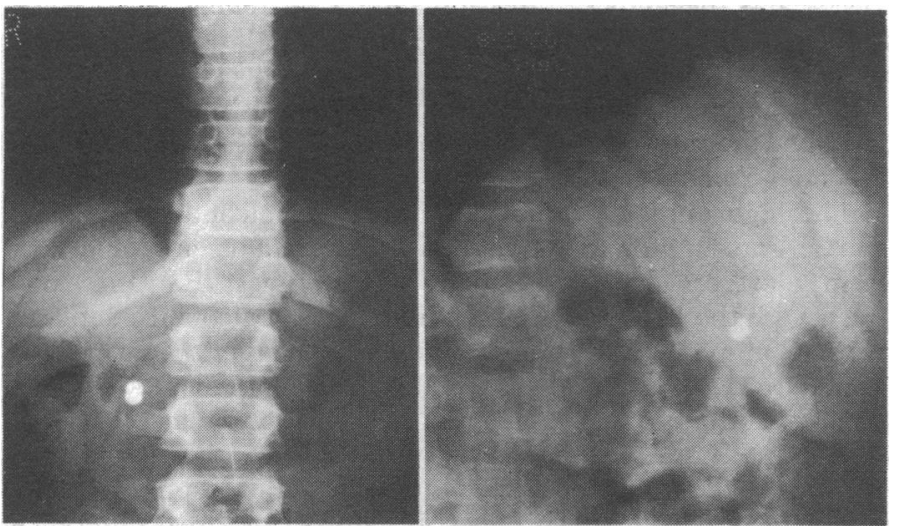

Anteroposterior and lateral radiographs showing pellet in upper abdomen.

At laparotomy the pellet track was found to pass obliquely through the abdominal wall and left lobe of the liver. The pellet had punctured the antrum and was lying within the lumen of the duodenum. The wounds were sutured and the pellet left to pass down the intestinal tract. The child made an uneventful recovery.

\section{Comment}

There have been few reports of air-weapon injuries, ${ }^{1-3}$ but all have emphasised their potentially serious nature. Most injuries are to the head, particularly the orbit, ${ }^{2}$ the thorax, and the extremities. There has been only one reported case of perforated viscus after abdominal injury. ${ }^{3}$

The belief that airguns are harmless and have little penetrating power is widespread among children and their parents. This is not the case. The pellet in our patient penetrated to a depth of about seven inches, and the weapon concerned was a rifle commonly bought for children.

The law restricts the sale of guns without a certificate to air weapons with a muzzle energy of less than $1.7 \mathrm{~kg} \mathrm{~m}$ (12 foot-pounds), ${ }^{4}$ but such an "unclassified" weapon can produce power far in excess of this. In the phenomenon of "dieseling"5 oil in the barrel is combusted by the heat generated by the passage of the pellet down the barrel The resultant explosion gives the pellet much greater velocity and unexpected penetrating power.

The law on air weapons restricts their use to children over the age of $14,{ }^{4}$ but such evidence as exists suggests that the age of the shooter in airgun accidents is commonly below this limit. ${ }^{1}$ It would seem pointless, therefore, to raise this age limit. There is currently nothing in law that requires the vendor of the air weapon to point out to the purchaser the potential dangers of the weapon or to advise him on a code of practice for its safe handling. As Bowen and Magauran $^{2}$ pointed out in 1973 a programme of education is needed for both children and adults. Nothing has yet been done, but as a start legislation is needed requiring that purchasers of air weapons are informed of the potential danger to prevent further needless injury.

I thank Mr J S Kirkham for his permission to report this case.

1 Reid IS. Airgun injuries in children. Med 7 Aust 1974 ;1:64-6.

2 Bowen DI, Magauran DM. Occular injuries caused by airgun pellets: an analysis of 105 cases. $\mathrm{Br} \mathrm{Med} \mathrm{F} \mathrm{1973;i:333-7.}$

${ }^{3}$ Rawson HD. The air rifle-a potentially lethal weapon. $N Z$ Med $\mathcal{f}$ $1965 ; 64: 327$.

4 House of Commons. Firearms Act. Section 53. London: HMSO, 1968. (Amended 1976.)

${ }^{5}$ Anonymous. Airgun injuries. Med f Aust 1974;1:55.

(Accepted 9 March 1981)

\section{St James's Hospital, Balham, London SW12}

ANDREW J G BATCH, BSC, FRCS, surgical registrar (present address: St George's Hospital, London SW17 0QT)

\section{Intractable pain in aplastic anaemia}

A patient with established aplastic anaemia developed recurrent episodes of intractable lumbar and leg pain. No clinical cause was ascertained. Necropsy confirmed the original diagnosis and provided evidence for a possible aetiology of pain.

\section{Case report}

A 32-year-old man was admitted in September 1977 with a history of increasing weakness, lethargy, and spontaneous bruising. Physical examination and laboratory investigations established a diagnosis of aplastic anaemia. No primary cause was identified. Conventional management with blood transfusion, anabolic steroids, and corticosteroids was satisfactory for 30 months.

In April 1980 he was admitted with severe lumbar and leg pain. On examination he was febrile and toxic and had distinct purpura with fundal haemorrhages. Neurological examination showed decreased pinprick perception below the level of T12. All other motor and sensory functions were normal. The severity of pain warranted continuous narcotic analgesia. Improvement occurred after a course of intravenous antibiotics and platelet transfusion. Complete remission of pain and neurological signs occurred within three weeks, but two weeks later a similar episode of pain necessitated readmission. Neurological examination showed hyperaesthesia below the level of T12. Investigations excluded leukaemic transformation and infectious causes. He was treated with intravenous antibiotics and narcotic analgesia; dexamethasone was given instead of prednisolone. Three further episodes were treated within three months without recourse to antibiotics or platelet transfusion. On each occasion neurological examination failed to identify a precise pattern of nerve distribution relevant to the symptomatology.

In July 1980 he died of congestive cardiac failure after an admission with a haemoglobin concentration of $4.8 \mathrm{~g} / \mathrm{dl}$. At necropsy there was no evidence of progression to leukaemia and the bone marrow was aplastic. Distinct haemosiderosis was present in all organs examined and in the marrow. Haemosiderin was found within the epineurium and perineurium of both posterior tibial nerves and within a sensory ganglion of the lumbar plexus. There was no demyelination or wallerian degeneration. Examination of the vertebral column showed no infective process, and the spinal cord showed no iron deposition or demyelination.

\section{Comment}

Clinical examination and laboratory investigations failed to identify the aetiology of the pain. At the conclusion of the first episode it was impossible to ascertain whether the antibiotics or platelet transfusion had induced resolution. In subsequent episodes first-line treatment with platelet transfusions was avoided as the patient had developed detectable antibodies. Remission of pain was, however, achieved with dexamethasone alone.

There was no evidence to substantiate a diagnosis of epidural abscess or leukaemic transformation. Episodic bone necrosis was 